\title{
MEASURES INVARIANT UNDER A LINEAR GROUP ${ }^{1}$
}

\author{
LARRY BAGGETT
}

\begin{abstract}
This paper deals with the question of when there exists on a Euclidean space a nontrivial probability measure $\mu$ which is invariant under a group $\Gamma$ of integer matrices. Necessary conditions on $\Gamma$ and the dimension are discussed. It is shown that nontrivial examples do exist, but only in dimensions $\geqslant 6$. In fact, the only explicit example given is in dimension 10 .
\end{abstract}

1. Introduction. Let $\Gamma$ be a group of invertible $n \times n$ (usually integer) matrices. We investigate the following: Under what conditions does there exist a nontrivial, $\Gamma$-invariant, Borel, probability measure on $\mathbf{R}^{n}$. The point mass at the origin is always invariant, so by "nontrivial" we shall mean a measure that is singular with respect to this point mass. We are also interested in the question of when there exists a faithful invariant measure $\mu$, by which we mean that, for each $\gamma$ in $\Gamma$, there exists a Borel set $E$ in $\mathbf{R}^{n}$ such that the $\mu$ measure of the symmetric difference between $E$ and $\gamma(E)$ is not 0 . Equivalently, $\mu$ is faithful if for every $\gamma$ in $\Gamma$ there exists a Borel set $E$ such that $\mu(E \cap \gamma(E)) \neq \mu(E)$. It is not clear a priori that invariant measures exist at all, and our first goal is to establish some necessary conditions on $n$ and $\Gamma$. Then, we give some examples, i.e., some sufficient conditions for their existence.

We are forced to extend slightly the notion of an "integer matrix". For a positive integer $q$, we let $\operatorname{SL}(n, \mathbf{Z} / q)$ denote the group of $n \times n$ matrices, of determinant 1 , each of whose entries is a rational number with denominator $\leqslant q$. We derive results for subgroups of this group rather than just those of $\operatorname{SL}(n, \mathbf{Z})$.

This work grew out of a problem in representation theory (see Theorem 2.6 below), and the author wishes to thank the harmonic analysts at the University of Rome for providing helpful sounding boards as this research evolved. In addition, this entire project resulted from the stimulation of Dennis Sullivan's visit to the University of Colorado in 1980.

\section{Some necessary conditions.}

2.1. Lemma. Let $A$ be an $n \times n$ real matrix, and let $\mu$ be an $A$-invariant Borel probability measure on $\mathbf{R}^{n}$. Then $\mu$ is supported on an A-invariant subspace $V$ of $\mathbf{R}^{n}$ such that the restriction of $A$ to $V$ belongs to a compact group of automorphisms of $V$.

Received by the editors June 20, 1984.

1980 Mathematics Subject Classification. Primary 22D10.

${ }^{1}$ This research was supported in part by a grant, MCS9301598, from the National Science Foundation. 
Proof. We may assume that the smallest $A$-invariant subspace of $\mathbf{R}^{n}$ which supports $\mu$ is all of $\mathbf{R}^{n}$. Let $p$ denote the Fourier transform of $\mu$, i.e., $p(z)=$ $\int e^{i(z, x)} d \mu(x)$. Then $p\left(A^{\prime}(z)\right)=p(z)$ for every $z$ in $\mathbf{R}^{n}$, where $A^{\prime}$ denotes the transpose of $A$. If $z$ is an eigenvector for $A^{\prime}$ with real eigenvalue $\lambda$, and if $|\lambda| \neq 1$, it follows that $p(t z) \equiv 1$ for all real $t$ (the orbit of $t z$ under $A^{\prime}$ has 0 as a limit point). This implies that $\mu$ is supported on the orthogonal complement to $z$ in $\mathbf{R}^{n}$. Similarly, if $A^{\prime}$ has a nonreal eigenvalue $\lambda$, with $|\lambda| \neq 1$, then there exists a two-dimensional subspace of $\mathbf{R}^{n}$ in which the orbit of any nonzero point has 0 as a limit point (the orbit being a spiral), and $\mu$ is again supported on the orthogonal complement to this subspace. Hence, we may assume that the eigenvalues of $A^{\prime}$ all have unit length.

Write $A^{\prime}=S+N$, where $S$ is semisimple, $N$ is nilpotent, and $S$ and $N$ commute. It will suffice to show that $N=0$, for then $A$ would be semisimple and have all its eigenvalues of unit length, whence it would be contained in a compact group. If $N$ is not 0 , we may choose a $z$ in $\mathbf{R}^{n}$ so that $N(z) \neq 0$, but $N^{2}(z)=0$. Then

$$
p(t z)=p\left(A^{\prime k}(t z)\right)=p\left((S+N)^{k}(t z)\right)=p\left(S^{k}(t z)+k t S^{k-1} N(z)\right)
$$

for all real $t$ and all positive integers $k$. We see from this, by taking $t$ small and $k$ appropriately, that for any positive numbers $r, s$, and $\varepsilon$, with $r<s$, there exists a $w$ in $\mathbf{R}^{n}$, with $r<\|w\|<s$, and $|1-p(w)|<\varepsilon$. Then it follows that for any positive $r$, there exists a $w$ in $\mathbf{R}^{n}$ with $\|w\|=r$ and $p(w)=1$. Hence, the additive subgroup $K$ of $\mathbf{R}^{n}$, consisting of the elements $w$ for which $p(w)=1$, is a closed and uncountable subgroup and therefore has positive dimension. However, $\mu$ is supported on the orthogonal complement to $K$, which implies that $\mu$ is supported on a proper subspace of $\mathbf{R}^{n}$, and this is a contradiction.

2.2. TheOREM. Let $\Gamma$ be a group of $n \times n$ real matrices, and let $\mu$ be $a \Gamma$-invariant, probability measure on $\mathbf{R}^{n}$. Suppose $V$ is the smallest subspace of $\mathbf{R}^{n}$ which supports $\mu$. Then

(i) $V$ is $\Gamma$-invariant.

(ii) The restriction of $\Gamma$ to $V$ is contained in a compact group $K$ of automorphisms of $V$.

In addition, if $\mu$ is ergodic, then

(iii) $\mu$ is the unique $K$-invariant measure on some $K$-orbit in $V$.

Proof. Part (i) follows because the intersection of two subspaces of measure 1 is again a subspace of measure 1. From Lemma 2.1 we know that the restriction to $V$ of each $\gamma$ in $\Gamma$ only has eigenvalues of unit length. If $K$ is defined to be the closure of the set of restrictions to $V$ of the elements in $\Gamma$, then it follows that the characteristic polynomial $\left(t-\lambda_{1}\right) \cdots\left(t-\lambda_{m}\right)$ of an $x$ in $K$ is the limit of characteristic polynomials $\left(t-\lambda_{1}^{j}\right) \cdots\left(t-\lambda_{m}^{j}\right)$ of elements in the restriction of $\Gamma$ to $V$. Hence, $x$ also only has eigenvalues of unit length. From the structure theory for Lie groups, we know that such a closed subgroup $K$ of matrices is necessarily compact. Finally, since the map $x \rightarrow \mu \circ x$ is continuous from the space of $n \times n$ matrices into the space of probability measures on $\mathbf{R}^{n}$, it follows that $\mu$ is $K$-invariant. Then (iii) is a classical result if $\mu$ is ergodic. 
2.3. TheOREM. Suppose $\Gamma$ is an infinite group of $n \times n$ integer matrices. Then no nontrivial $\Gamma$-invariant measure exists if

(i) $\Gamma$ acts irreducibily on $\mathbf{R}^{n}$ (no proper invariant subspaces);

(ii) $\Gamma$ contains an element each of whose eigenvalues is of nonunit length;

(iii) $\Gamma$ is $\operatorname{SL}(n, Z / q)$ for $q$ a positive integer.

Proof. (i) and (ii) follow immediately from the preceding lemma and theorem. (iii) is true because $\operatorname{SL}(n, Z / q)$ contains elements each of whose eigenvalues has nonunit length.

REMARK. Let $\Gamma$ be a group of $n \times n$ matrices for which a nontrivial $\Gamma$-invariant probability measure exists. Then there exists a basis of $\mathbf{R}^{n}$ in which each element of $\Gamma$ has the block form $\left[\begin{array}{ll}A & B \\ 0 & C\end{array}\right]$, where the $A$ 's belong to a compact group of automorphisms of a $\Gamma$-invariant subspace $V$ (the smallest subspace supporting $\mu$ ). If $\mu$ is faithful, then the map $\gamma \rightarrow A$ is one-to-one, which implies that $\Gamma$ is algebraically isomorphic to a subgroup of a compact Lie group. Conversely, suppose $\mu$ is not faithful and $\gamma$ is an element of $\Gamma$ for which $\mu(E \cap \gamma(E))=\mu(E)$ for every Borel set $E$. It follows that if $x$ is an element of $\mathbf{R}^{n}$ for which $\gamma(x) \neq x$, then there exists a neighborhood $U$ of $x$ with $\mu(U)=0$. Hence, $\mu$ is supported on the set of $\gamma$-fixed vectors in $\mathbf{R}^{n}$. Because $V$ is the smallest subspace supporting $\mu$, we see that the matrix $A$ corresponding to $\gamma$ is the identity. Therefore, $\mu$ is faithful if and only if $\gamma \rightarrow A$ is one-to-one. As a consequence of this observation, we have

2.4. Proposition. If $\Gamma$ is solvable, and if a faithful $\Gamma$-invariant probability measure exists, then $\Gamma$ is finite modulo a torus.

More specifically, we have

2.5. Proposition. If $\Gamma$ is a discrete, infinite, cyclic subgroup of $\operatorname{SL}(3, \mathbf{R})$, then no faithful $\Gamma$-invariant probability measure exists on $\mathbf{R}^{3}$.

Proof. Let $\gamma$ be a generator for $\Gamma$, and suppose such a $\mu$ exists. Represent $\gamma$ in block form as $\left[\begin{array}{ll}A & B \\ 0 & C\end{array}\right]$ according to the above remark. The matrix $A$ cannot be $3 \times 3$, for then the infinite discrete group $\Gamma$ would be contained in a compact group. The matrix $A$ cannot be $1 \times 1$, for then it would be \pm 1 , and $\mu$ would not be faithful. Therefore, $A$ must be a $2 \times 2$ matrix with eigenvalues $\lambda_{1}$ and $\bar{\lambda}_{1}$ with $\left|\lambda_{1}\right|=1$. But then the matrix $C$ is $1 \times 1$ and must in fact equal $1(\operatorname{det}(\gamma)=1)$. The characteristic polynomial for $\gamma$ is then of the form $(x-1)\left(x^{2}+b x+1\right)$. Hence,

$$
\lambda_{1}=\left(-b \pm\left(b^{2}-4\right)^{1 / 2}\right) / 2 \text {, }
$$

and since $\left|\lambda_{1}\right|=1$, we have $b= \pm 2$, whence $\lambda_{1}= \pm 1$; but again $\mu$ would not be faithful, so the proof is complete.

Definition. A locally compact group $G$ is said to have Kazhdan's property $T$ if the trivial representation of $G$ is an isolated point in the unitary dual of $G$. See [1].

A relationship between property $T$ and the existence of invariant measures is the following:

2.6. THEOREM. Let $\Gamma$ be a group of $n \times n$ matrices, and let $G$ be the semidirect product $\mathbf{R}^{n} * \Gamma$. Then $G$ has property $\mathrm{T}$ if and only if $\Gamma$ has property $\mathrm{T}$ and there exists no nontrivial $\Gamma$-invariant probability measure on $\mathbf{R}^{n}$. 
Proof. Suppose $G$ has property T. Then the quotient group $\Gamma$ has property $\mathrm{T}[\mathbf{1}]$. If there is a nontrivial $\Gamma$-invariant probability measure $\mu$ on $\mathbf{R}^{n}$, define a sequence $\left\{\mu_{n}\right\}$ of measures $\mu_{n}$ by $\mu_{n}(E)=\mu(n E)$. Then $\mu_{n}$ also is nontrivial and $\Gamma$-invariant, and $\left\{\mu_{n}\right\}$ converges weakly to the point mass at 0 . If $\pi_{n}$ denotes the unitary representation of $G$ defined on $L^{2}\left(\mu_{n}\right)$ by

$$
\left[\pi_{n_{(x, y)}}(f)\right](y)=e^{i(x, y)} f\left(\gamma^{-1}(y)\right),
$$

and if we take $v_{n}$ to denote the constant function 1 in $L^{2}\left(\mu_{n}\right)$, then $\left(\left[\pi_{n_{(x, y)}}\left(v_{n}\right)\right], v_{n}\right)$ converges to 1 uniformly on compact subsets of $G$, which implies that the trivial representation of $G$ is not isolated.

Conversely, suppose $\Gamma$ has property $\mathrm{T}$ but $G$ does not. Let $\left\{\pi_{n}\right\}$ be a sequence of nontrivial, irreducible, unitary representations of $G$, and let $\left\{v_{n}\right\}$ be a sequence of unit vectors for which the functions $\left(\left[\pi_{n_{(x, y)}}\left(v_{n}\right)\right], v_{n}\right)$ converge uniformly on compacta to 1. Such sequences can be found if the trivial representation is not isolated. From Mackey theory, there must exist a sequence $\left\{\nu_{n}\right\}$ of probability measures such that $\pi_{n}$ is equivalent to the representation $\rho_{n}$, acting in $L^{2}\left(\nu_{n}, K_{n}\right)\left(K_{n}\right.$ a Hilbert space), defined by the formula

$$
\left[\rho_{n_{(x, \gamma)}}(f)\right](y)=p(y, \gamma) e^{i(x, y)} R_{n}(y, \gamma)\left(f\left(\gamma^{-1}(y)\right)\right),
$$

where $p$ is a scalar function (the square root of a certain Radon-Nikodym derivative), and $R_{n}$ is a "cocycle" for the action of $\Gamma$ on $\mathbf{R}^{n}$ with coefficients in the unitary group of $K_{n}$.

Because $\Gamma$ has property $\mathrm{T}$, we know that for large $n$ there exists a function $f_{n}$ in $L^{2}\left(\nu_{n}, K_{n}\right)$ which is $\Gamma$-invariant, i.e.,

$$
p(y, \gamma) R_{n}(y, \gamma)\left(f_{n}\left(\gamma^{-1}(y)\right)\right)=f_{n}(y)
$$

for all $\gamma$ in $\Gamma$. Taking norms, we find that

$$
p(y, \gamma)\left\|f_{n}\left(\gamma^{-1}(y)\right)\right\|=\left\|f_{n}(y)\right\|,
$$

which implies that the Radon-Nikodym cocycle $p$ is a coboundary, whence $\nu_{n}$ is equivalent to a finite $\Gamma$-invariant measure $\mu_{n}$. This measure is nontrivial, for otherwise $\pi_{n}$ would be the trivial representation. This completes the proof.

REMARK. A completely analogous argument shows that a general semidirect product $N * H$ has property $\mathrm{T}$ if and only if $H$ does and there does not exist a sequence $\left\{\mu_{n}\right\}$ of nontrivial $H$-invariant probability measures on the unitary dual $\hat{N}$ which converges weakly to the point mass at the trivial representation of $N$.

As a consequence of the preceding theorem, we deduce

2.7. Proposition. Let $\Gamma$ be a "lattice" subgroup of $\operatorname{SL}(n, \mathbf{R})$. Then no nontrivial $\Gamma$-invariant measures exist on $\mathbf{R}^{n}$.

PROOF. For $n \leqslant 2$ this follows from Theorem 2.3. Since, for $n>2$, all such lattices (discrete subgroups of cofinite volume) have property $\mathrm{T}$, we may prove this result, using Theorem 2.6 , by verifying that $\mathbf{R}^{n} * \Gamma$ has property $\mathrm{T}$. But, using Theorem 2.6 in the other direction and Theorem 2.3 , we know that $\mathbf{R}^{n} * \operatorname{SL}(n, \mathbf{R})$ has property $\mathrm{T}$, whence so does $\mathbf{R}^{n} * \Gamma$, it being a subgroup of $\mathbf{R}^{n} * \operatorname{SL}(n, \mathbf{R})$ with cofinite volume. 
2.8. TheOREM. Let $q$ be a positive integer, let $\Gamma$ be a subgroup of $\operatorname{SL}(n, Z / q)$, and suppose that $\mu$ is a faithful $\Gamma$-invariant, probability measure on $\mathbf{R}^{n}$. Let $V$ be the smallest subspace of $\mathbf{R}^{n}$ which supports $\mu$, and represent each element $\gamma$ of $\Gamma$ in block form as $\left[\begin{array}{ll}A & B \\ 0 & C\end{array}\right]$, where $A$ belongs to a fixed compact group $K$ of transformations of $V$. Then

(i) if $\Gamma$ is nonamenable, $n \geqslant 6$;

(ii) if $\Gamma$ has property $\mathrm{T}, n \geqslant 7$;

(iii) if $\operatorname{dim} V$ is odd, and $\Gamma$ has property $\mathrm{T}$, the matrices $C$ are at least $5 \times 5$ and $n \geqslant 8$.

Proof. The kernel of the homomorphism $\gamma \rightarrow \operatorname{det}(A)$ is a subgroup of finite index in $\Gamma$, so we may replace $\Gamma$ by this subgroup and assume, without loss of generality, that $\operatorname{det}(A) \equiv 1$. If $\Gamma$ is nonamenable, then $\operatorname{dim} V \geqslant 3$. Also, the map $\gamma \rightarrow C$ is a homomorphism of $\Gamma$ whose kernel $M$ is a discrete subgroup of the group $N$ consisting of all $n \times n$ matrices of the form $\left[\begin{array}{cc}A & B \\ 0 & I\end{array}\right]$, where $A$ belongs to $K$ and $B$ is arbitrary. This group $N$ is the semidirect product of an abelian group (the $B$ 's) and the compact group $K$, whence $N$ and $M$ are both amenable. Hence, if $\Gamma$ is nonamenable, then so is the group of $C$ 's, whence the $C$ 's must be at least $2 \times 2$. Therefore, if $\operatorname{dim} V \geqslant 4$, part (i) is clear.

If $\operatorname{dim} V=3$, then each matrix $A$ has 1 as an eigenvalue. It then follows from Lemma A below that each matrix $C$ also has 1 as an eigenvalue. If the $C$ 's were only $2 \times 2$, it would follow that 1 is the only eigenvalue for each $C$. But then the group of $C$ 's would be unipotent, hence amenable. Therefore, if $\operatorname{dim} V$ is 3 , then the $C$ 's are at least $3 \times 3$, and this completes the proof of part (i).

Similarly, if $\Gamma$ has property $\mathrm{T}$, then the group of $C$ 's must have property $\mathrm{T}$, so they must be at least $3 \times 3$. This establishes (ii) when $\operatorname{dim} V \geqslant 4$. Of course, (iii) will imply the rest of (ii), and (iii) is a consequence of Lemmas A and B below.

LEMMA A. Let $n$ be the smallest integer for which there is a positive integer $q$ and a nonamenable subgroup $\Gamma$ of $\operatorname{SL}(n, Z / q)$ such that a faithful, $\Gamma$-invariant, probability measure $\mu$ exists on $\mathbf{R}^{n}$. Let $V$ be the smallest (proper) subspace of $\mathbf{R}^{n}$ which supports $\mu$, and represent the elements $\gamma$ of $\Gamma$ in block form $\left[\begin{array}{ll}A & B \\ 0 & C\end{array}\right]$, where $A$ has determinant 1 and belongs to a compact group of automorphisms of $V$. Let $W$ denote the set of fixed vectors in $\mathbf{R}^{n}$ for an element $\gamma$ of $\Gamma$, and assume that $W \cap V$ is nontrivial. Then $W$ is not contained in $V$. In particular, if the matrix $A$ has 1 as an eigenvalue, then the corresponding matrix $C$ has 1 as an eigenvalue.

Proof. We argue first that, by minimality of $n$, the group $\Gamma$ cannot leave invariant any proper subspace of $\mathbf{Q}^{n}$. Indeed, if $S$ is a proper, $\Gamma$-invariant subspace of $\mathbf{Q}^{n}$ which spans an $m$-dimensional subspace $T$ of $\mathbf{R}^{n}$ (with $m<n$ ), let $\phi_{1}$ be the homomorphism which sends an element of $\Gamma$ to its restriction to the subspace $T$, and let $\phi_{2}$ be the homomorphism which sends an element of $\Gamma$ to its projection onto the quotient space $\mathbf{R}^{n} / T$. Let $\mu_{1}$ denote the measure on $T$ obtained by restricting $\mu$, and let $\mu_{2}$ be the projection of $\mu$ onto the quotient space. One sees that at least one of the pairs $\left(\phi_{i}(\Gamma), \mu_{i}\right)$ satisfies the hypotheses of the lemma and acts in a space of smaller dimension. Therefore, no such $\Gamma$-invariant subspace $S$ of $\mathbf{Q}^{n}$ can exist. 
Now, the subspace $W$ is spanned by vectors with rational coordinates (being the range of a transformation of the form $p(\gamma)$ for some rational polynomial $p$ ). Hence, the $\Gamma$-invariant subspace $W^{\prime}$, generated by $W$ under $\Gamma$, contains a nonzero $\Gamma$ invariant subspace of $\mathbf{Q}^{n}$. If $W$ is contained in the proper, $\Gamma$-invariant subspace $V$, then so is $W^{\prime}$. But this implies that $\Gamma$ leaves invariant a proper subspace of $\mathbf{Q}^{n}$.

LEMMA B. Let $\Gamma$ be a noncompact closed subgroup of $\operatorname{SL}(n, \mathbf{C})$ and suppose $\Gamma$ has property T. Assume that each element of $\Gamma$ has 1 as an eigenvalue. Then $n \geqslant 5$.

Proof. Let $G$ denote the algebraic closure (over the complex field) of $\Gamma$, i.e., $G$ is the set of all $n \times n$ matrices $M$ such that whenever a polynomial $p$ satisfies $p(\gamma)=0$ for every $\gamma$ in $\Gamma$ then $p(M)=0$. This algebraic closure $G$ is a group, whence an algebraic group containing a noncompact closed subgroup $\Gamma$ having property T. If $S$ is the Levy factor of $G$, then $S$ must be nontrivial, and must also contain a noncompact closed subgroup which has property T. Similarly, the commutator subgroup $L$ of $S$ is a semisimple Lie group containing a noncompact closed subgroup having property $T$.

If $p$ is the polynomial $\operatorname{det}(M-1)$, then $p(\gamma)=0$ for every $\gamma$ in $\Gamma$, so that $\operatorname{det}(M-1)=0$ for every $M$ in $L$. The lemma now reduces to finding the minimal dimension $n$ for which there exists a connected semisimple Lie group $L$, containing a noncompact closed subgroup having property $\mathrm{T}$, such that each element of $L$ has 1 as an eigenvalue. An examination of the list of classical simple Lie groups and their "small" representations shows that dimension 5 is the smallest.

3. Examples. We see from Theorem 2.5 that dimension 4 is the smallest dimension in which an infinite group $\Gamma$ of integer matrices can possibly have a faithful invariant measure.

3.1. EXAMPLE. Let $A$ be the $4 \times 4$ matrix

$$
\left[\begin{array}{cccc}
0 & 0 & 0 & -1 \\
1 & 0 & 0 & -3 \\
0 & 1 & 0 & 0 \\
0 & 0 & 1 & -3
\end{array}\right]
$$

with characteristic (minimal) polynomial $x^{4}+3 x^{3}+3 x+1$. One shows by calculus that this polynomial has exactly two real zeroes and, hence, two complex zeroes. Also, if $\lambda$ is a root, than so is $1 / \lambda$, so $A$ is represented in an appropriate basis by the matrix

$$
\left[\begin{array}{cccc}
\cos (\alpha) & \sin (\alpha) & 0 & 0 \\
-\sin (\alpha) & \cos (\alpha) & 0 & 0 \\
0 & 0 & \lambda & 0 \\
0 & 0 & 0 & 1 / \lambda
\end{array}\right]
$$

where $\alpha$ is not a rational multiple of $2 \pi$ because $e^{i \alpha}$ is a root of the above characteristic equation which no root of unity can satisfy. Lebesgue measure on any circle in the plane spanned by the first two coordinate vectors in this basis will clearly be a faithful invariant measure on $\mathbf{R}^{4}$ for the group generated by $A$. 
REMARK. In general, we find it difficult to construct groups of integer matrices for which faithful invariant measures exist. The most interesting result we have found is the following:

3.2. THEOREM. There exists a positive integer $q$ and a subgroup $\Gamma$ of $\operatorname{SL}(n, Z / q)$ for which a faithful, $\Gamma$-invariant, probability measure exists on $\mathbf{R}^{n}$, such that

(i) $\Gamma$ has property $\mathrm{T}$,

(ii) $n \leqslant 10$.

Proof. We begin by recalling the group $G$ defined by Sullivan in [2]. Let $B$ be the quadratic form on $\mathbf{R}^{10}$ defined by

$$
x_{1}^{2}+x_{2}^{2}+x_{3}^{2}+2^{1 / 2} x_{4}^{2}+2^{1 / 2} x_{5}^{2}+x_{6}^{2}+x_{7}^{2}+x_{8}^{2}-2^{1 / 2} x_{9}^{2}-2^{1 / 2} x_{10}^{2},
$$

and let $H$ be the group of $10 \times 10$ matrices $\left[\begin{array}{ll}A & 0 \\ 0 & A^{\prime}\end{array}\right]$, where $A$ preserves the positive definite form represented by the $5 \times 5$ matrix

$$
\left[\begin{array}{cc}
I_{3} & 0 \\
0 & 2^{1 / 2} I_{2}
\end{array}\right]
$$

and where $A^{\prime}$ preserves the indefinite form represented by the matrix

$$
\left[\begin{array}{cc}
I_{3} & 0 \\
0 & -2^{1 / 2} I_{2}
\end{array}\right]
$$

( $I_{m}$ denoting the $m \times m$ identity matrix). Sullivan's group $G$ is the subgroup of $H$ consisting of those matrices in $H$ whose entries belong to the ring $\mathbf{Z}\left[2^{1 / 2}\right]$ for which $A^{\prime}=\sigma(A)$, where $\sigma$ is the automorphism of $\mathbf{Z}\left[2^{1 / 2}\right]$ defined by $\sigma\left(2^{1 / 2}\right)=-2^{1 / 2}$.

Sullivan showed that $G$ is a lattice in $H$ (and hence has property $\mathrm{T}$ ) and the map $g \rightarrow A$ is an isomorphism onto a dense subgroup of the "orthogonal" group $K$ preserving that positive definite form. Let $G^{\prime}$ denote this isomorphic copy of $G$ in $K$.

Next, let $N$ denote the additive subgroup of $\mathbf{R}^{5}$ consisting of the vectors whose coordinates belong to the ring $\mathbf{Z}\left[2^{1 / 2}\right]$, and let $L$ denote the semidirect product $N * G^{\prime}$ ( $G^{\prime}$ leaves $N$ invariant). Obviously, $L$ is dense in the semidirect product $\mathbf{R}^{5} * K$. Since this latter group fails to have property $\mathrm{T}$ (Theorem 2.6 if nothing else), it follows that $L$ fails to have property T. But $N$ is isomorphic to $\mathbf{Z}^{10}$, and $G^{\prime}$ is then a group of automorphisms of $\mathbf{Z}^{10}$, and so is isomorphic to a group $\Gamma$ of $10 \times 10$ integer matrices. Hence, the countable group $L$ is isomorphic to the semidirect product $\mathbf{Z}^{10} * \Gamma$, which is a discrete subgroup of cofinite volume in the semidirect product $\mathbf{R}^{10} * \Gamma$.

Since $L$ fails to have property $\mathrm{T}$, it follows that $\mathbf{R}^{10} * \Gamma$ also fails to have property T. Then, by Theorem 2.6, there must exist a nontrivial $\Gamma$-invariant measure $\mu$ on $\mathbf{R}^{10}$, and we let $V$ denote the smallest subspace of $\mathbf{R}^{10}$ which supports $\mu$. If $\mu$ is not faithful, let $J$ denote the subgroup of $\Gamma$ consisting of the $\gamma$ 's for which the $\mu$ measure of the symmetric difference of $\gamma(E)$ and $E$ is 0 for every Borel set $E$. If $W$ denotes the intersection, over all $\gamma$ in $J$, of the subspaces of $\gamma$-fixed vectors, then $W$ is a proper $\Gamma$-invariant subspace (because $J$ is normal). Further, $W$ contains $V$ and is spanned by vectors having rational coordinates with denominators bounded by a 
positive integer $q$. But then $\Gamma$ would leave invariant a proper subspace of $\mathbf{Q}^{10}$, in which case we could reduce (as in the proof of Lemma A) to an example of even lower dimension. This completes the proof.

REMARK 1 . We cannot verify that the $\Gamma$ of the preceding proof acts irreducibly on $\mathbf{Q}^{10}$, although it seems likely. If it acts reducibly, i.e., there exists a discrete subgroup $\Gamma$ of $\operatorname{SL}(9, Z / q)$ which has property $\mathrm{T}$ and for which there is a faithful $\Gamma$-invariant probability measure, the arguments of $\$ 2$ can be used to show that there exists a dense subgroup of $\mathrm{SO}(3)$ with property $\mathrm{T}$ and, therefore, to prove the uniqueness of rotationally invariant, finitely additive measures on the 2-sphere. See [2].

REMARK 2. Sullivan's construction can be performed in all dimensions $n \geqslant 2$, not just $n=5$. The corresponding groups $G$ fail to have property $\mathrm{T}$ for $n<5$, so that we cannot prove, using the above arguments, the existence of invariant measures for the corresponding $\Gamma$ 's, Theorem 2.6 no longer being applicable. It seems likely, however, that $\Gamma$-invariant measures do exist for all $n>1$ for these $\Gamma$ 's.

\section{REFERENCES}

1. D. Kazhdan, On a connection of the dual space of the group and the structure of its closed subgroups, Functional Anal. Appl. 1 (1967), 71-74.

2. D. Sullivan, For $n>3$ there is only one finitely additive rotationally invariant measure on the $n$-sphere defined on all Lebesgue measurable sets, Bull. Amer. Math. Soc. (N.S.) 4 (1981), 121-123.

Department of Mathematics, University of Colorado, Boulder, Colorado 80309 